\title{
Bemerkungen zur Veränderlichkeit und Streuung von Seegangsbeobachtungen im Flachwasserbereich
}

\author{
H. Walden, K. Rrchter \& H. Carlson \\ Deutsches Hydrograpbisches Institut; \\ Hamburg 4, Bundesrepublik Deutschland
}

\begin{abstract}
Remarks on variability and scattering of wave observations in shallow water areas. Wave data, obtained by measurements off the coast of the Federal Republic of Germany scatter very strongly, even within "classes" of uniform wind conditions present at measuring time. The reasons for the considerable scatter are enumerated and discussed. The method used by GIENAPP (1972) for deriving wave properties from strong winds by extrapolation is criticized. It is proposed to conduct wave measurements only during selected, defined weather situations.
\end{abstract}

\section{EINLEITUNG}

Seit 1961 stellt das Deutsche Hydrographische Institut mit Hilfe eines Schwimmergerätes Messungen des Seegangs an verschiedenen Positionen dicht vor den deutschen Küsten an. Meßergebnisse wurden hauptsächlich vom DeUTSCHEN HYorograpHsCHEN Insti'tuT (DHI) (1972), Interpretationen z. B. von G. ToMczaK (1969) und GIENAPP $(1967,1972)$ veröffentlicht. Die Meßmethode und die Art der Auswertung hat u. a. SCHRADER (1968) beschrieben.

Die genannten Arbeiten befassen sich in der Hauptsache mit der Beziehung zwischen den Seegangseigenschaften und dem Wind. Hierzu wurde der Wind in 16 Richtungssektoren und die Beaufort-Stärken 0 bis 12 eingeteilt. Man erhält also $16 \times 13=$ 208 Windstärke-Windrichtungsklassen (kurz: „Windklassen“).

Ein Blick in die statistischen Aufstellungen des DHI (1972a, b) lehrt, daß die Wellenhöhen außerdem von dem gerade herrschenden Wasserstand abhängen. Die sich hieraus ergebende starke Aufteilung der vorliegenden Meßdaten hat zur Folge, daß viele Windklassen schwach oder gar nicht mit Wellenbeobachtungen besetzt sind.

\section{ANGABEN UBER DIE STREUUNG DER DATEN}

Betrachten wir aus den Angaben des DHI (1972b) willkürlich ausgewählte Ausschnitte; sie sind der ersten und dritten Tabellenseite entnommen (Tab. 1). Die statistisch unzureichende Besetzung bewirkt, daß bestimmte Einzelwerte auffällig aus der zu erwartenden Verteilung herausfallen. 
Tabelle 1

Ausschnitte aus Tabellen des Deutschen Hydrographischen Instituts (1972b)

\begin{tabular}{|c|c|c|c|c|c|c|c|}
\hline \multirow{2}{*}{$\begin{array}{l}\text { a) Station } \\
\text { Wind- } \\
\text { stärke } \\
\text { (Bft) }\end{array}$} & \multicolumn{3}{|c|}{$\begin{array}{c}\text { Scharhörn-Riff (NNE-Wind) } \\
\text { Zahl der }\end{array}$} & \multirow{2}{*}{$\begin{array}{l}\text { b) Station } \\
\text { Wind- } \\
\text { stärke } \\
\text { (Bft) }\end{array}$} & \multicolumn{3}{|c|}{$\begin{array}{l}\text { Scharhörn-Riff (ENE-Wind } \\
\text { Zahl der }\end{array}$} \\
\hline & $\begin{array}{l}\text { Wasser- } \\
\text { stand }\end{array}$ & $\begin{array}{l}\text { Fälle } \\
\text { (2 Min. } \\
\text { Registrie- } \\
\text { rung) }\end{array}$ & $\mathrm{H}_{\text {Mittel }}$ & & $\begin{array}{l}\text { Wasser- } \\
\text { stand }\end{array}$ & $\begin{array}{l}\text { Fälle } \\
\text { (2 Min. } \\
\text { Registrie- } \\
\text { rung) }\end{array}$ & $\vec{H}_{\text {Mittel }}$ \\
\hline 1 & $\begin{array}{l}<5 \mathrm{~m} \\
5-6,5 \\
>6,5\end{array}$ & & & 1 & $\begin{array}{l}<5 \mathrm{~m} \\
5-6,5 \\
>6,5\end{array}$ & & \\
\hline 2 & $\begin{array}{l}<5 \mathrm{~m} \\
5-6,5 \\
>6,5\end{array}$ & $\begin{array}{l}1 \\
2\end{array}$ & $\begin{array}{l}44 \\
31\end{array}$ & 2 & $\begin{array}{l}<5 \mathrm{~m} \\
5-6,5 \\
>6,5\end{array}$ & $\begin{array}{l}1 \\
1\end{array}$ & $\begin{array}{l}13 \\
25\end{array}$ \\
\hline 3 & $\begin{array}{l}<5 \mathrm{~m} \\
5-6,5 \\
>6,5\end{array}$ & $\begin{array}{l}6 \\
5\end{array}$ & $\begin{array}{l}37 \\
42\end{array}$ & 3 & $\begin{array}{l}<5 \mathrm{~m} \\
5-6,5 \\
>6,5\end{array}$ & $\begin{array}{l}3 \\
6\end{array}$ & $\begin{array}{l}20 \\
26\end{array}$ \\
\hline 4 & $\begin{array}{l}<5 \mathrm{~m} \\
5-6,5 \\
>6,5\end{array}$ & $\begin{array}{r}9 \\
10 \\
1\end{array}$ & $\begin{array}{l}31 \\
43 \\
13\end{array}$ & 4 & $\begin{array}{l}<5 \mathrm{~m} \\
5-6,5 \\
>6,5\end{array}$ & $\begin{array}{r}8 \\
12 \\
1\end{array}$ & $\begin{array}{l}29 \\
31 \\
62\end{array}$ \\
\hline 5 & $\begin{array}{l}<5 \mathrm{~m} \\
5-6,5 \\
>6,5\end{array}$ & $\begin{array}{r}20 \\
8\end{array}$ & $\begin{array}{l}50 \\
53\end{array}$ & 5 & $\begin{array}{l}<5 \mathrm{~m} \\
5-6,5 \\
>6,5\end{array}$ & $\begin{array}{r}26 \\
21 \\
1\end{array}$ & $\begin{array}{l}35 \\
38 \\
46\end{array}$ \\
\hline 6 & $\begin{array}{l}<5 \mathrm{~m} \\
5-6,5 \\
>6,5\end{array}$ & $\begin{array}{l}3 \\
2\end{array}$ & $\begin{array}{l}52 \\
74\end{array}$ & 6 & $\begin{array}{l}<5 \mathrm{~m} \\
5-6,5 \\
>6,5\end{array}$ & $\begin{array}{l}25 \\
20\end{array}$ & $\begin{array}{l}48 \\
59\end{array}$ \\
\hline 7 & $\begin{array}{l}<5 \mathrm{~mm} \\
5-6,5 \\
>6,5\end{array}$ & & & 7 & $\begin{array}{l}<5 \mathrm{~m} \\
5-6,5 \\
>6,5\end{array}$ & $\begin{array}{l}4 \\
3\end{array}$ & $\begin{array}{l}54 \\
72\end{array}$ \\
\hline 8 & $\begin{array}{l}<5 \mathrm{~m} \\
5-6,5 \\
>6,5\end{array}$ & & & 8 & $\begin{array}{l}<5 \mathrm{~m} \\
5-6,5 \\
>6,5\end{array}$ & & \\
\hline
\end{tabular}

Der Ausschnitt von der ersten Seite, Scharhörn-Riff, NNE-Wind (Tab. 1a), enthält z. B. einen "Ausschießer“ des auf einer einzigen Beobachtung beruhenden Wertes für die Wellenhöhe bei Windstärke 4 und Wasserstand $>6,5 \mathrm{~m}$. Mit $13 \mathrm{~cm}$ ist er der kleinste Wert dieser Spalte überhaupt, d. h. kleiner als $\bar{H}_{\text {Mittel }}$ bei den Windstärken 2 und 3 .

Auf der dritten Seite, Scharhörn-Riff, ENE-Wind (Tab. 1b), erscheint für $\overline{\mathrm{H}}_{\text {Mittel }}$ bei $4 \mathrm{Bft}$ und Wasserstand $>6,5$ ein Wert von $62 \mathrm{~cm}$; er fällt an dieser Stelle erheblich nach oben heraus. Allerdings handelt es sich hier gleichfalls um ein „Kollektiv“ der Größe 1 (es ist nur ein einziger Meßwert vorhanden). Ahnliche Fälle lassen sich in dem Beobachtungsmaterial in großer Zahl finden.

$\overline{\mathrm{H}}_{\text {Mittel }}$ ist das arithmetische Mittel aus sämtlichen in der betreffenden Windklasse zur Verfügung stehenden $\overline{\mathrm{H}}$-Werten, wobei $\overline{\mathrm{H}}$ die mittlere Wellenhöhe während einer einzelnen, sich tiber eine bestimmte Meßzeit erstreckende Seegangsbeobachtung 


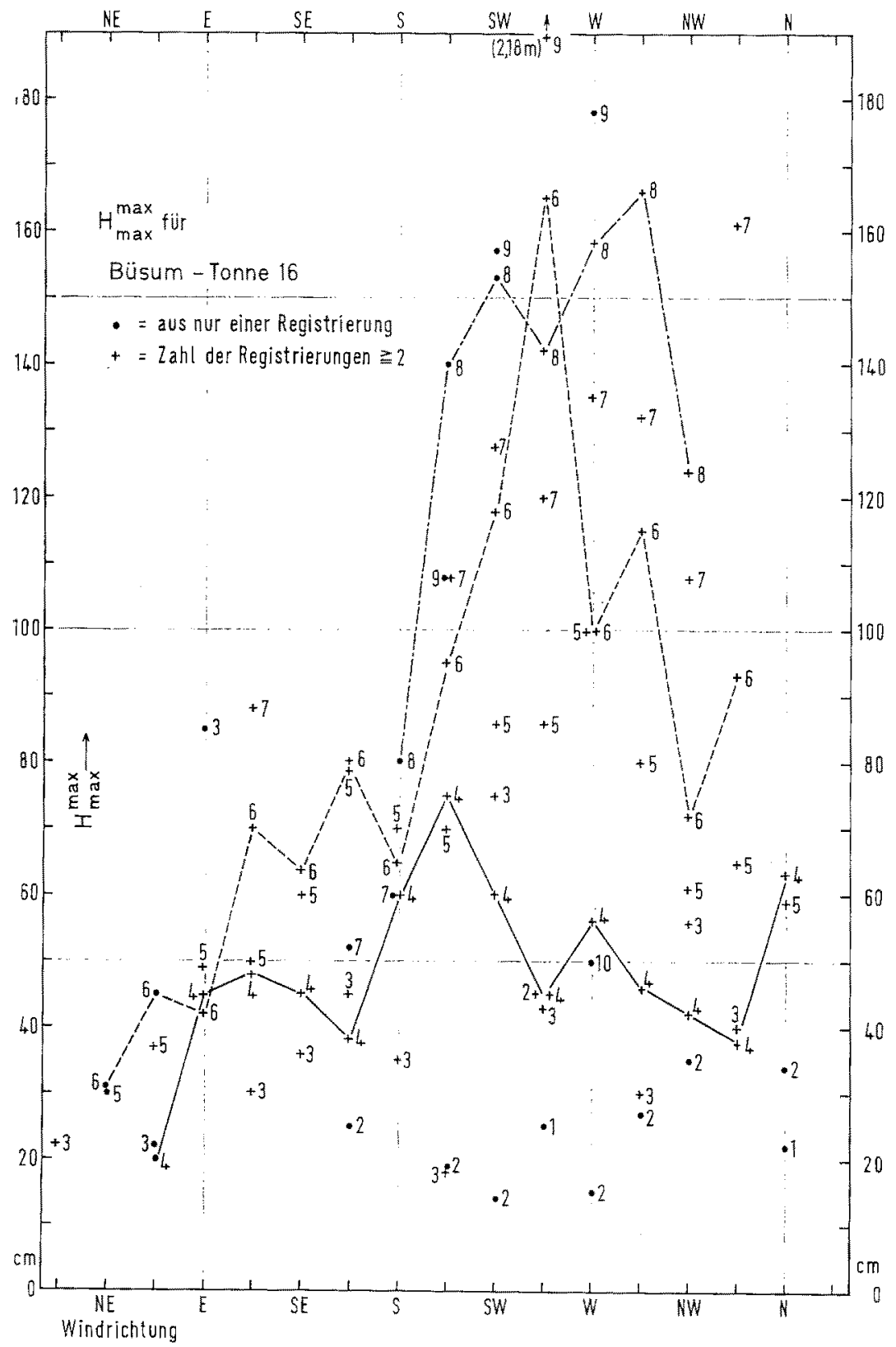

Abb. 1: $\mathrm{H}_{\max }^{\max }$ für Büsum-Tonne 16 bei verschiedenen Windrichtungen und -stärken. Es bedeuten: - dem eingetragenen Wert liegt nur eine Registrierung zugrunde; + Zahl der Registrierungen $\geqq 2$; beigefügte Ziffer (z. B. +4 ) bezeichnet die Beaufort-Windstärke 
bedeutet. Betrachten wir für die Windklasse Südwest $5^{*}$ die einzelnen für die Meßstationen "Tonne 16“ (vor Büsum) vorliegenden $\overline{\mathrm{H}}$-Werte; eingeteilt nach 3 Wasserstandsbereichen W:

$$
\begin{aligned}
& \text { für } W<5 \mathrm{~m}: \quad \overline{\mathrm{H}}=27,17,20,24,20,19,31,26,27,20,24,25,23,22,14 \mathrm{~cm} \\
& \text { für } W>5-6,5 \mathrm{~m}: \quad=29,21,21,35,30,15,32,25,26,21,34,31,23,32,28,15 \text {, } \\
& 21,29,19,24,11,28,22,26,26,21,22,13 \mathrm{~cm} \\
& \text { für } W>6,5 \mathrm{~m} \quad 28,24,32,30,29,20,26,31,24,21 \mathrm{~cm}
\end{aligned}
$$

Für $\mathrm{W}=5-6,5 \mathrm{~m}$ schwanken die erfaßten $\overline{\mathrm{H}}-\mathrm{Werte}$ also $\mathrm{zwischen} 13 \mathrm{~cm}$ und $35 \mathrm{~cm}$.

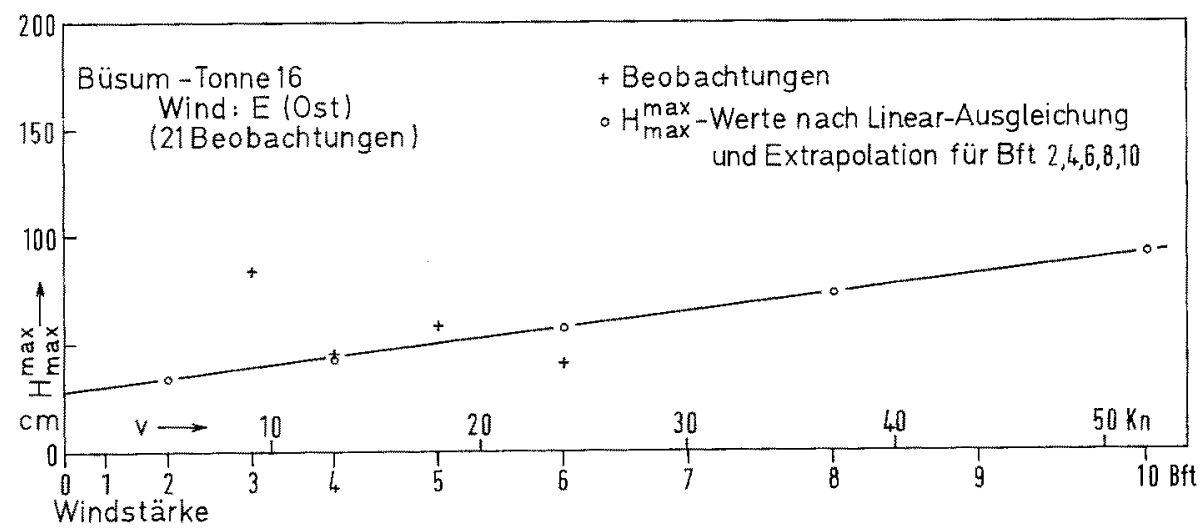

Abb. 2: $H_{\max }^{\max }$ für Büsum-Tonne 16 bei Ostwind für verschiedene Beaufort-Windstärken. Es bedeuten: + Beobadhtungen; $\circ \mathrm{H}_{\max }^{\mathrm{max}}$-Werte nach linearer Ausgleichung und Extrapolation für Bft 2, 4, 6, 8 und 10 (gemäß Grenapp 1972)

Aus der großen Streuung ergibt sich, daß auch die $\overline{\mathrm{H}}_{\text {Mittel }}$-Werte eine große $\mathrm{Zu}$ fallsabhängigkeit besitzen. Sie ist um so größer, je kleiner das Beobachtungs-Kollektiv der betreffenden Windklasse ist.

Die meereskundlichen Beobachtungen und Ergebnisse Nr. 32 und Nr. 33 (DHI 1972a, b) enthalten auch Angaben über die pro Windklasse jemals ermittelten größten Einzelwellen. (Während die $\overline{\mathrm{H}}$-Werte aus Registrier-"Blöcken“ von unterschiedlicher Dauer - $1 / 2$ bis $2^{1 / 2}$ Std. je nach Seegangshöhe - ermittelt wurden, sind diese $\mathrm{H}_{\max }{ }^{-}$ Werte den zwischengeschalteten "Schnellschrieben" von je zwei Minuten Dauer entnommen worden.) $\mathrm{H}_{\max }^{\max }$ bezeichnet die größte Wellenhöhe, die bei allen vorliegenden Registrierungen in der betreffenden Windklasse erfaßt wurde. Da es sich um Einzelwerte handelt, sind die $\mathrm{H}_{\max }^{\max }$-Werte sehr streuungs- und zufallsempfindlich.

Die Abbildung 1 enthält für die Station Büsum „Tonne 16“ eine graphische $\mathrm{Zu}$ sammenstellung der $\mathrm{H}_{\text {max }}^{\max }$-Werte, wie sie in den Tabellen (DHI 1972a) aufgeführt sind.

* Für diese Windklasse ist die Zahl der Beobachtungen groß im Vergleich zu anderen Klassen. 
Die durch + und $\bullet$ gekennzeichneten Eintragungen geben den $\mathrm{H}_{\max }^{\max }$-Wert für die betreffende Windrichtung bei der in Ziffern beigefügten Windstärke wieder, z. B. +4 für $4 \mathrm{BA}$; + bezieht sich auf Fälle, in denen $\geqq 2$ Registrierungen vorliegen. Der Eintragung $\bullet$ liegt eine einzige ausgewertete Registrierung zugrunde. Für 4, 6 und $8 \mathrm{Bft}$ sind die Eintragungen zur besseren Erkennbarkeit der Verteilung durch unterschiedlich markierte Linien verbunden.

Man erkennt, daß in einer Anzahl von Fällen $\mathrm{H}_{\max }^{\max }$ nicht entsprechend der $\mathrm{Zu}$ nahme der Windstärke größer wird. Diese Erscheinung ist die Folge der Streuung der Meßgrößen; ihre Ursachen sind oben genannt worden. Außerdem zeigt die Abbildung, daß für Windstärken $>8 \mathrm{Bft}$ nur für wenige Richtungen Meßgrößen von $\mathrm{H}_{\max }^{\max }$ vor-

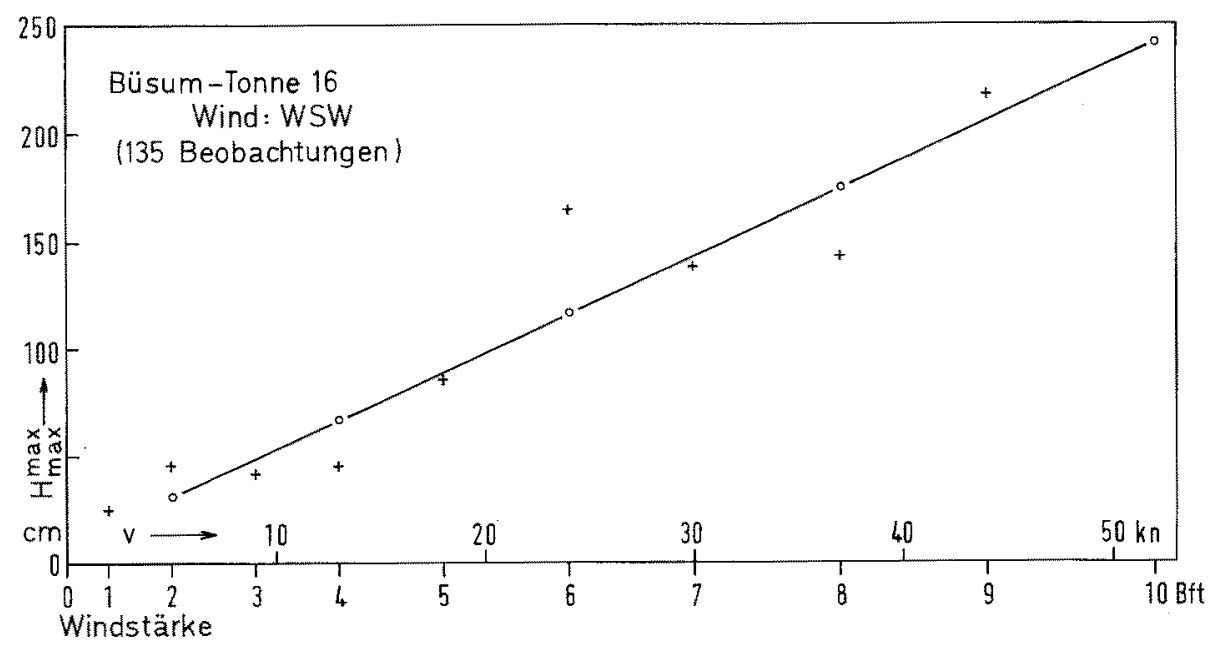

Abb. 3: $\mathrm{H}_{\max }^{\max }$ für Büsum-Tonne 16 bei WSW-Wind für verschiedene Windstärken. Es bedeuten: + Beobachtungen; $\circ \mathrm{H}_{\max }^{\max }$-Werte nach linearer Ausgleichung und Extrapolation für BAt 2, $4,6,8$ und 10 (gemäß GIENAPP 1972)

liegen und sogar Bft 8 nur bei den Richtungen S, SSW, SW, WSW, W, WNW und NW durch Beobachtungen belegt ist; der einzige Wert für $10 \mathrm{BA}$ liegt trotz auflandiger Windrichtung bei nur $50 \mathrm{~cm}$ !

$\mathrm{Da} B$ die entsprechenden Darstellungen in früheren Veröffentlichungen (GIENApp 1972) ein ganz anderes Bild ( $\mathrm{vgl}$. dort Abb. 2a) bieten, beruht auf einer speziellen Behandlung des Materials, die hier kurz geschildert werden soll.

Unsere Abbildungen 2, 3 und 4 enthalten für je e in e Windrichtung (Ost, WSW und NNW) die für die verschiedenen Windstärken ermittelten $\mathrm{H}_{\max }^{\max }$-Werte. Nachdem die als Kreuz gezeichneten Beobachtungswerte je nach Größe des Kollektivs mit Gewichten (auch das nicht beobachtete $\mathrm{H}_{\max }^{\max }=0$ für $\mathrm{O} \mathrm{Bft}$ ) versehen waren, wurde für die Darstellung in Gienapp (1972) eine mittlere gerade Linie nach der Methode der kleinsten Quadrate berechnet. Die entsprechende Gleichung für die Gerade wurde (arithmetisch) linear extrapoliert, so daß man für die großen Windstärken $\mathrm{H}_{\max }^{\max }$ Werte ermitteln konnte. Bei diesen Größen, die in Abbildung 2, 3 und 4 durch o ge- 
kennzeichnet sind, handelt es sich also nicht um Beobachtungen, sondern um Zahlen, die nach einem wegen der geringen Zahl der pro Klasse vorliegenden Beobachtungen anfechtbaren Verfahren abgeleitet wurden. Wegen der großen Streuung der Beobachtungen unterliegt die Neigung der Geraden Zufälligkeiten, die zu wirklich fiktiven Extrapolationswerten führen. In den Abbildungen 2, 4 und 5 von GrenapP (1972) sind alle Werte auf solche Geraden bezogen; nur deshalb verlaufen die Polygonzüge dort in einer verblüffenden Ordnung, die tatsächlich nur durch Ausgleichsmaßnahmen erreicht

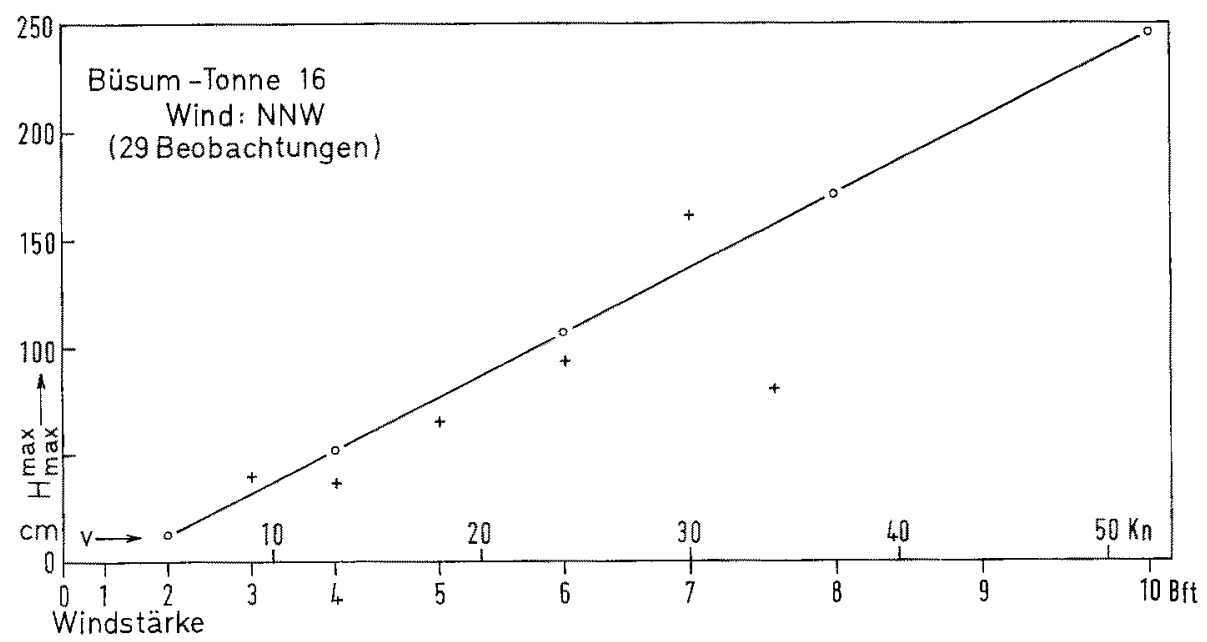

Abb. 4: $H_{\max }^{\max }$ für Büsum-Tonne 16 bei NNW-Wind für verschiedene Windstärken. Es bedeuten: + Beobachtungen; $\bigcirc \mathrm{H}_{\max }^{\mathrm{max}}-W$ Werte nach linearer Ausgleichung und Extrapolation für Bft $2,4,6,8$ und 10 (gemäß GIENAPp 1972)

wurde. Solche Geraden stellt GIENAPp (1972) in seiner Abbildung 3 selbst dar, nur erfährt der Leser dort nicht, wie die Werte zustande gekommen sind.

Die Wellenhöhen, und zwar sowohl $\bar{H}$ als auch $H_{\max }^{\max }$, sprechen stark auf die Windrichtung an; es folgt dies aus der unterschiedlichen Streichlänge über See. Auch Untiefen in Streichrichtung können die Entwicklung des Seegangs behindern (vgl. z. B. GIENAPP 1972). In den hier behandelten Fällen geht aber ein großer Teil der $z$. T. groBen Unterschiede von $\overline{\mathrm{H}}$ und $\mathrm{H}_{\max }^{\max }$ zwischen benachbarten Windvektoren mit Sicherheit auf die Kleinheit der Beobachtungskollektive, die große Streuung und somit auf den Zufall zurück (in Abb. 2a ist GIENAPp bei NNW ein Fehler im Sinne zu kleiner $\mathrm{H}_{\max }^{\max }$-Werte unterlaufen, der ihn auch der Sorge enthebt, dieses größte aller Maxima zu erklären; der Wert für $10 \mathrm{Bft}$ beträgt $246 \mathrm{~cm}$ statt ca. $196 \mathrm{~cm}$ in Abb. 2a von GIENAPP 1972).

\section{URSACHEN FUR DIE GROSSE STREUUNG}

Es wurden die Standardabweichung ("Streuung") und der Variabilitätskoeffizient für $\overline{\mathrm{H}}$ nach den üblichen Formeln (vgl. z. B. Henrysson et al. 1960): 


$$
\sigma=\sqrt{\frac{\sum\left(\overline{\mathrm{H}}-\overline{\mathrm{H}}_{\mathrm{Mittel}}\right)^{2}}{\mathrm{n}-1}} \text { und } \mathrm{v}=\sqrt{\frac{\sum\left(\overline{\mathrm{H}}-\overline{\left.\mathrm{H}_{\text {Mittel }}\right)^{2}}\right.}{\mathrm{n}-1}} \frac{100}{\overline{\mathrm{H}}}
$$

( $\mathrm{n}=$ Zahl der Fälle pro Windklasse)

für eine Anzahl von Windklassen berechnet, von denen einige wiedergegeben seien:

\begin{tabular}{|c|c|c|c|c|c|c|c|}
\hline \multirow{4}{*}{ "Tonne $16^{4}$} & & & SW 4 & SW 5 & SW 6 & SW 7 & \\
\hline & $W<5 \mathrm{~m}$ & $\begin{array}{l}\sigma= \\
v=\end{array}$ & $\begin{array}{c} \pm 3,1 \\
22\end{array}$ & $\begin{array}{c} \pm 4,3 \\
19\end{array}$ & $\begin{array}{c} \pm 4,5 \\
15\end{array}$ & $\begin{array}{c} \pm 1,7 \\
5\end{array}$ & $\%$ \\
\hline & $W=5-6,5 \mathrm{~m}$ & $\begin{array}{l}\sigma= \\
V=\end{array}$ & $\begin{array}{c} \pm 5,7 \\
34\end{array}$ & $\begin{array}{c} \pm 6,2 \\
26\end{array}$ & $\begin{array}{c} \pm \quad 6,5 \\
21\end{array}$ & $\begin{array}{c} \pm 6,4 \\
16\end{array}$ & $0 \%$ \\
\hline & $W>6,5 \mathrm{~m}$ & $\begin{array}{l}\sigma= \\
\mathrm{v}=\end{array}$ & & $\begin{array}{c} \pm 4,3 \\
16\end{array}$ & $\begin{array}{c} \pm 4,7 \\
13\end{array}$ & $\begin{array}{c} \pm 5,3 \\
13\end{array}$ & $\%$ \\
\hline
\end{tabular}

Diese hohen Werte der Streuungsgrößen dürften in der Hauptsache auf folgende Einflüsse zurïckzuführen sein: (a) Unterschiedliche Dauer des der Aufstellung zugrunde gelegten Windes; die See war nicht in allen Fällen in bezug auf den Wind voll entwickelt bzw. unterschiedlich weit entwickelt; (b) Windfeld unterschiedlicher Länge und Breite; die See war bei kleinem Fetch nicht voll entwickelt (kommt im wesentlichen nur bei auflandigen Winden in Betracht); (c) Dünung, in der Regel aus einer anderen als der Windrichtung und zum Teil von fern anlaufend, kann die lokale Windsee überlagert und daher die Gesamt-Wellenhöhe vergrößert haben; (d) die Böigkeit des Windes beeinflußt die Entwicklung des Seegangs; (e) Strömungen, insbesondere Gezeitenströme, können Einfluß auf den Seegang ausgeübt haben.

(a), (b) und (c) ließen sich auch so zusammenfassen: Der am Ort und zur Zeit der Seegangsbeobachtung herrschende Wind ist nicht allein maßgebend für die Eigenschaften des örtlichen (windbedingten) Seegangs.

\section{KONSEQUENZEN}

Der Zweck von Dauerstationen für Seegangsmessungen ist es, das örtliche „Seegangsklima " oder "Seegangsregime“ kennenzulernen. Gemeint ist die örtliche, dünungsfreie Windsee. $\mathrm{Da}$ in der Hauptsache die maximalen Eigenschaften des Seegangs von Interesse sind, würde es genügen, solche Beobachtungen für statistische Aufstellungen heranzuziehen, für die Winddauer und Größe des Windfeldes (über See und Land) so groß sind, daß sie die Entwicklung des Seegangs nicht behindern. Diese Fälle kann man nur mit Hilfe von Wetterkarten ausfindig machen. So sollte es gelingen, die Streuung der Meßwerte weitgehend auszuschalten.

Für dieses Vorgehen benötigt man ein umfassendes Beobachtungsmaterial (verwendbare Beobachtungen möglichst für jede der 160 Windklassen bei Windstärken 1-10 Bft). Man muß also an e in er Station sehr lange beobachten, um möglichst viele geeignete Wetterlagen zu erfassen.

Wesentlich effektiver wäre es, Messungen nur dann anzustellen, wenn die Forde- 
rungen der Wetterlage erfüllt sind. Diese Möglichkeit besteht, wenn man die Meßgeräte über Funk ein- und ausschaltet.

Man muß sich aber im klaren darüber sein, daß in einem Seebereich, in dem Küstenformation und Topographie des Meeresgrundes sehr unruhig sind - wie dies an den deutschen Küsten der Fall ist -, die Beziehung zwischen Wind und Seegang an einer Meßstation und entsprechend eine Statistik des örtlichen Seegangsregimes nur für ein kleines Gebiet repräsentativ sind.

\section{ZUSAMMENFASSUNG}

1. Betrachtet werden Wellenmessungen mit Schwimmergeräten an Meßpfählen vor den deutschen Küsten. Die nach Beobachtungsstellen und in Klassen annähernd einheitlicher Windrichtung und -stärke geordneten Wellengrößen unterliegen einer sehr starken Streuung. Dies wird an Beispielen gezeigt, wobei Gründe angegeben werden.

2. Mittelwerte pro „Windklasse“ streuen gleichfalls, weil die Zahl der Beobachtungen pro Klasse in den meisten Fällen zu gering ist.

3. Kritisiert wird eine von GrENAPP (1972) verwendete Methode; mit ihr wurden für Windklassen, für die keine Wellenbeobachtungen vorliegen, Wellengrößen durch Ausgleichung und Extrapolation abgeleitet.

4. Schließlich wird vorgeschlagen, nur Messungen bei ausgewählten Wetterlagen auszuwerten bzw. in Zukunft nur bei bestimmten, ausgewählten Windsituationen zu messen.

\section{ZITIERTE LITERATUR}

Deutsches HydrograpHusches Instrtut, 1972a. Seegangsmessungen in der Piep (Deutsche Bucht). Meeresk. Beob. Ergebn. 32.

- 1972b. Seegangsmessungen in der Elbe. Meeresk. Beob. Ergebn. 33.

GreNAPP, H., 1967. Vergleich berechneter und gewonnener Seegangsgrößen von Mellum Plate (Deutsche Bucht). Dt. hydrogr. Z. 20, 64-72.

- 1972. Wellenmessungen im Seegebiet der Piep (Deutsche Bucht). Helgoländer wiss. Meeresunters, $23,261-267$.

Henrysson, S., Haseloff O. W. \& Hofmann, H. J., 1960. Kleines Lehrbuch der Statistik. De Gruyter, Berlin, 173 pp.

Schrader, J. P., 1968. Kennzeichnende Seegangsgrößen für drei Meßpunkte in der Elbemündung. Hamburger Küstenforsch. 4, 1-76.

TomczaK, G., 1969. Bericht über mittlere Wellenhöhen und -perioden der Seegangsmeßstationen Westerland, Büsum, Tertius, Wangerooge, Mellum Plate und Außeneider. Int. Schifffahrtskongr. (Paris) 22, 286-295.

Anschrift des erstgenannten Autors: $\mathrm{H}$. WALDEN

Deutsches Hydrographisches Institut

2 Hamburg 4

Bernhard-Nocht-Straße 78

Bundesrepublik Deutschland 